\title{
Layered-Division Multiplexing in 5G New -Radio for Terrestrial Broadcast Services
}

\author{
MadhusmitaShial $^{\mathrm{a}}$, SatyasisMishra $^{\mathrm{b}} *$ \\ ${ }^{a} \mathrm{Ph} . \mathrm{D}$. Scholar, Centurion University of Technology \& Management, Odisha, India \\ ${ }^{\mathrm{b}}$ Dept.Of.ECE, Centurion University of Technology \& Management, Odisha, India \\ amadhusmitha.shial@gmail.com, b.mishra@cutm.ac.in \\ b*Corresponding author: s.mishra@cutm.ac.in
}

Article History: Received: 10 November 2020; Revised 12 January 2021 Accepted: 27 January 2021; Published online: 5 April 2021

\begin{abstract}
The 5G system can deliver simultaneouslyunicast services, multicast services and broadcast services using the conventional high-power high- tower (HPHT) infrastructure and with the same spectrum.The Third Generation Partnership Project (3GPP) LTEevolved -multimedia broadcast- multicast service (eMBMS) have new features designed for Terrestrial Broadcast services in Release 14. A broadcast service means only receive mode, only downlink no uplink or inserting a SIM card not needed. In free to air reception without a SIM card or without user device registration with the network the content can be received. This specification is standardized in release 15. In this study a two layer Layered-Division Multiplexing (LDM) is used in 5G new radio (NR).A significant performance could be analyzed in a 5G- NRMBMS by using two layer LDM system for delivering terrestrial broadcast services. The performance analysis demonstrated by computer simulations. Using two-layer LDM in 5G-NRMBMS system a high-qualityservice can be provided to both handheld and fixed devices
\end{abstract}

Keywords: broadcast, 5G NR, eMBMS,LDM, layers, Terrestrial, services

\section{Introduction}

Within 2017 to 2020 the data signal traffic increases by seven-fold and more than 80 percent data signal traffic for high definition (HD) videos. So, presently it's a great challenge for broadcasters and mobile operators to catch up to this exponentially growing data demands [1]. This high data traffic puts more pressure on mobile operators to allocate more spectrums and to use the latest technologies to improve channel efficiency. The 5G technology uses millimeter wave (mmWave) spectrum even though it is challenging to transmit ultra-highdefinition (UHD) quality video to a greater number of users simultaneously. So, it is important now to integrate both unicast and broadcast in a single system. Most popular programs or sports which more users are likely to view at the same time can be broadcast. In this scenario 5G network can deliver broadcast services, IoT, public safety, emergency alert systems, connected vehicles and terrestrial TV[1].

LTE 'enhanced Multicast/Broadcast Multimedia Service'(eMBMS) mobile broadcast services already included. For $5 \mathrm{G}$ broadcasting the architecture introduces receive -only mode, without SIM and without uplink. Basic functions of multicast/broadcast services for 5G-NR architecture may be announced in release 17 [2]. In 3GPP release-6 the first multimedia multicast broadcast service (MBMS) system introduced then in release-14 the evolved multimedia multicast broadcast service '(eMBMS)' systemdeveloped for 4G/LTE. Then 'further evolved multi-mediamulticast broadcast service’ (FeMBMS) system was introduced in release-14 with some new functions to deliver terrestrial broadcast services. Further $200 \mu \mathrm{sec}$ of cyclic-prefix (CP)and $1.25 \mathrm{kHz}$ subcarrier spacing added to get high-spectral efficiency[1]. In release-16 the cyclic prefix will have at least $300 \mu$ sec and core 'OFDM' symbol duration of $2.4 \mathrm{~ms}$ minimum. In terrestrial broadcasting $100 \%$ of NR carriers may be occupied to fixed, mobile or handheld services only in downlink mode. The NRMBMS configuration is more flexible to provide different network types from single cell to nationwide SFN and from fixed rooftop to high speed mobile reception [3].

In this study the performance analysis will evaluate by using two-layer LDM in 5G New Radio MBMS (5G NRMBMS). The Section-II architecture of Two-layer LDM, Section III describes the 5G NR physical Layer, Section-iv the performance analysis for two layer LDM for 5G-NRMBMS and finally the paper is concluded.

\section{Architecture of Two Layer LDM}

A two-layer LDM transmitter and receiver block diagram is shown in Figure:-1. 


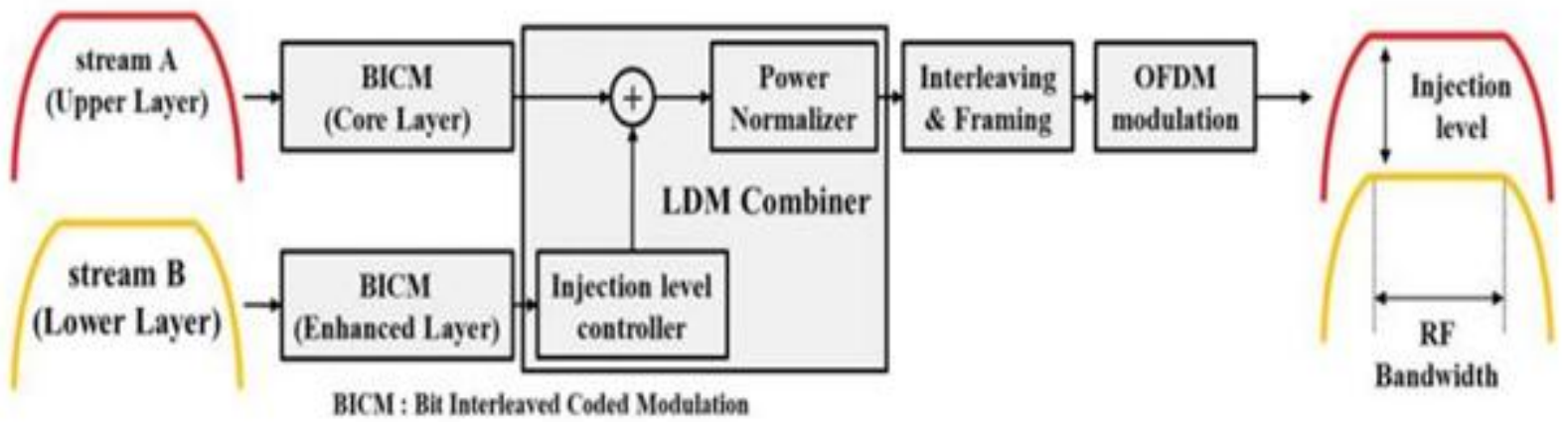

Fig .1 Two layer LDM Transmitter block diagram [4]

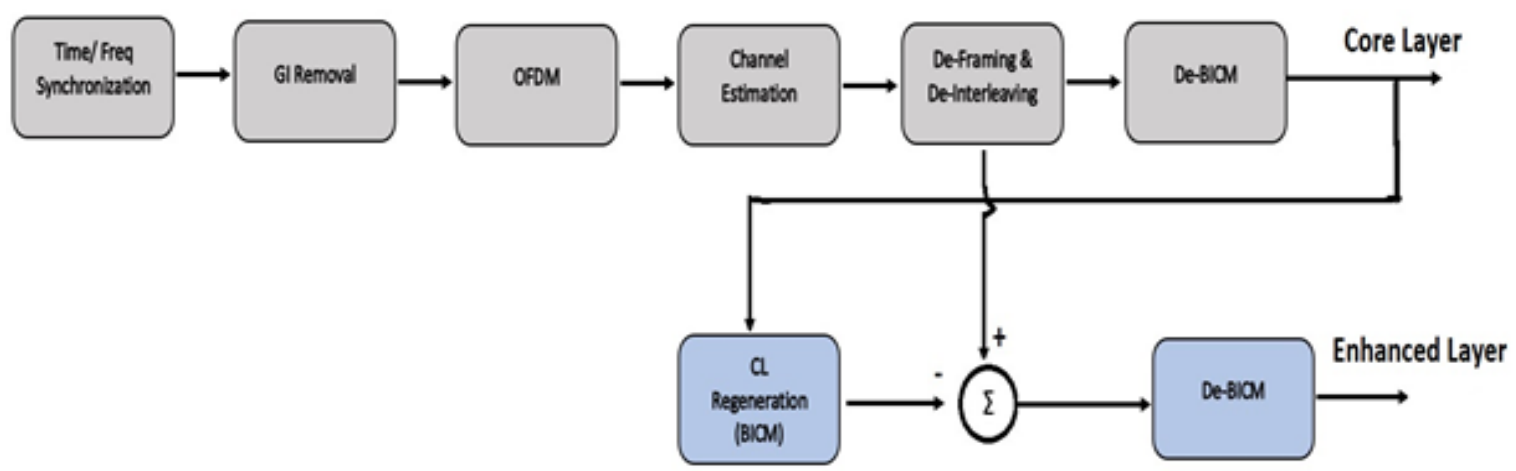

Fig 2 Two layer LDM receiver Block diagram [5]

Layered-Division-Multiplexing (LDM) is a power based non orthogonal multiplexing (P-NOM) technology. LDM is a layered transmission structure, used to transmit multiple signal layers with different power levels simultaneously. LDM uses $100 \%$ of RF bandwidth and time resources for better spectrum efficiency. Figure 1 shows transmitter blocks of 2-layer LDM, in which different layers are assigned with different transmission power and with different sets of channel mapping, channel coding interleaving etc [1]. Different transmission signals share the same 'OFDM' structure. The OFDM signal includes FFT size, cyclic prefix (CP) duration and pilot insertion. In Figure2 shows the receiver of two-layer LDM first- layer (core layer) is the higher Power level and the second layer (enhanced layer) is the lower power level signal. At receiving end, the first layer signal will decode first treating the second layer signal as interference. When second layer signal will decode, first layer signal will decode and cancelled then second layer signal detection carried out [6].

\section{5G NR PHYSICAL LAYER}

The 3GPP New Radio rel-15 and rel-16 focused on only unicast but after RAN\#79 meeting the broadcast work distributed into two groups, one is mixed unicast/multicast/broadcast and other one is LTE-based 5G Terrestrial broadcast. 5G-NR provides a more efficient way of design than an LTE in the case of extensive frequency bands, use cases and deployments. In $5 \mathrm{G}-\mathrm{NR}$, the resource grid contains subcarriers and it represent in frequency domain. The symbol represents in time domain. The resource grid consists of a combination of Resource Blocks (RB) and one RB containing 12 subcarriers in frequency domain. The waveforms are combined with scalable numerology. The sub-carrier spacing (SCS) scaled in $15 \mathrm{KHz} \times 2^{\mu}$, where $\mu=0$ to 4 and $15 \mathrm{KHz}$ is the base SCS. There are five SCS- $15 \mathrm{KHz}, 30 \mathrm{KHz}, 60 \mathrm{KHz}, 120 \mathrm{KHz}$ and $240 \mathrm{KHz}$. In 5G-NR a slot contains 14 OFDM symbols always. It configures $100 \mathrm{MHz}$ or $400 \mathrm{MHz}$ bandwidth carriers. The $5 \mathrm{G}-\mathrm{NR}$ brings a new concept known as Carrier-Bandwidth-Part (CBP), by using CBP a group of consecutive resource blocks can be defined with different numerology ofthe same carrier. Maximum of four CBP can be given by which different multiplexing and different numerologies can be used from a single wideband transmitter [7] .The 5G-NR physical channel and signals with downlink and uplink are given in Table-1 [10]. 
Table: -1 5G-NR physical Channel and Signals [10]

\begin{tabular}{|c|c|c|c|}
\hline \multicolumn{2}{|l|}{ Down Link } & \multicolumn{2}{|l|}{ Up Link } \\
\hline DL Physical Channels & DL Physical Signals & UL Physical Channels & UP Physical Signal \\
\hline $\begin{array}{l}\text { Physical DL control } \\
\text { Channel (PDCCH) }\end{array}$ & $\begin{array}{l}\text { Primary Synchronization } \\
\text { Signal (PSS) }\end{array}$ & $\begin{array}{l}\text { Physical UL Control } \\
\text { Channel (PUCCH) }\end{array}$ & $\begin{array}{l}\text { Sounding Reference Signal } \\
\text { (SRS) }\end{array}$ \\
\hline $\begin{array}{l}\text { Physical DL Shared } \\
\text { Channel (PDSCH) }\end{array}$ & $\begin{array}{l}\text { Secondary Synchronization } \\
\text { Signal (SSS) }\end{array}$ & $\begin{array}{l}\text { Physical UL Shared } \\
\text { Channel (PUSCH) }\end{array}$ & $\begin{array}{l}\text { Phase Tracking } \\
\text { Signal (PTRS) }\end{array}$ \\
\hline $\begin{array}{l}\text { Physical Broadcast } \\
\text { Channel }(\mathrm{PBCH})\end{array}$ & $\begin{array}{l}\text { Channel State inf- } \\
\text { Reference Signal (CSI-RS) }\end{array}$ & $\begin{array}{lr}\begin{array}{l}\text { Physical } \\
\text { Access }\end{array} & \text { Random- } \\
\text { (PRACH) } & \\
\end{array}$ & $\begin{array}{l}\text { Demodulation } \\
\text { Signal (DMRS) }\end{array}$ \\
\hline & $\begin{array}{l}\text { Phase Tracking Reference } \\
\text { Signal (PTRS) }\end{array}$ & & \\
\hline & $\begin{array}{l}\text { Demodulation References } \\
\text { Signal (DMRS) }\end{array}$ & & \\
\hline
\end{tabular}

Table 2: 5G-NR Channels and Signals[7]

\begin{tabular}{|c|c|c|}
\hline Bandwidth & SHCCH & From 4.32 to $400 \mathrm{MHz}$ \\
\hline \multirow{5}{*}{ Type of coding } & PDSCH & LDPC \\
\hline & $\mathrm{PDCCH}$ & Polar \\
\hline & $\mathrm{PBCH}$ & Polar \\
\hline & PUSCH & LDPC \\
\hline & PUCCH & Polar/Block \\
\hline \multirow{8}{*}{ Type of SS sequences } & PSS & $\mathrm{m}$-sequence \\
\hline & SSS & Gold sequence \\
\hline & DMRS & Gold sequence \\
\hline & CSI-RS & Gold sequence \\
\hline & PTRS & Gold sequence \\
\hline & SRS & Gold sequence \\
\hline & PRACH & m-sequence \\
\hline & PUCCH & ZC(e.g.ACK/NACK) \\
\hline \multirow{5}{*}{ Type of modulation } & PDSCH & Up to 256QAM \\
\hline & $\mathrm{PDCCH}$ & QPSK \\
\hline & $\mathrm{PBCH}$ & QPSK \\
\hline & PUSCH & $\begin{array}{llll}\pi / 2-\mathrm{BPSK} & \text { and } & \mathrm{Up} & \text { to } \\
256 \mathrm{QAM}\end{array}$ \\
\hline & PUCCH & BPSK/QPSK \\
\hline
\end{tabular}

In Table-2 the 5G-NR physical channel and signals shown, where SHCCH is shared and control channel. The 5G-NR frame structure, one frame consists of 10 sub-frames. $N_{\text {frame }}=10$ sub-frame, each sub-frame is of $1 \mathrm{~ms}$ and sub-frames consistof a flexible number of slots. Each slot consists of 14 or 12 OFDM symbols. For Normal $\mathrm{CP}$ it is 14 symbols and for extended CP it is 12 symbols.5G- NR configure higher system bandwidth carriersi.e $100 \mathrm{MHz}$ or $400 \mathrm{MHz}$. PTRS a new reference-signal introduced in 5G-NR for Time or Frequency tracking and DMRS (Demodulation -Reference-Signals) is used for channel-estimation. LDPC (Low-Density-Parity-Check) adopted for user plane data .channel polarization concept a new technique is used for channel control and this 5GNR polar code giving better performance than LTE Tail-Biting- Convolutional -Codes(TBCC)[7]. In 5G NR irrespective of SCS a slot always contains 14 'OFDM' symbols therefore the number of slots varies with SCS.

For designing 5G NR terrestrial broadcast a set of numerologies considered in [7].When $\mu=0$ is suitable for terrestrial broadcast in SFN or MFN mode and $\mu$ with negative factor can be used for SFN operation with extended CP for LPLT deployment. But in broadcast services large cells for HPHT deployment this is impractical. In $5 \mathrm{G}-\mathrm{NR} 15 \mathrm{KHz} \mathrm{SCS}$ is the standard numerology with $25 \mathrm{RBs}$ per $5 \mathrm{MHz}$. 


$$
S C_{R B}=12.15 \mathrm{KHz} \times T_{S} \times(1-C P)
$$

From the above equation a multiple combination of ' $\mathrm{T}_{\mathrm{CP}}$ ' and ' $\mathrm{T}_{\mathrm{U}}$ ' can be derived. The number of ' $\mathrm{SC}$ ' per 'RB' can be derived by means of OFDM symbol-duration and CP. The CP and OFDM symbol-duration should be selected in manner to get integer numbers of SC per RB. According to thetransmitting and receiving environment the possible combination should select. For better mobility performance the possible numerologies to select. Decreasing of $\mathrm{T}_{\mathrm{CP}} / \mathrm{T}_{\mathrm{U}}\left(\mathrm{T}_{\mathrm{U}}=\right.$ OFDM symbol duration \& " $\mathrm{T}_{\mathrm{CP}}=\mathrm{CP}$ " duration) ratio decay the ' $\mathrm{CP}$ ' overhead but increasing FFT size will cause receiver complexity[7].

For broadcasting in 5G-NR the FR1 range " $5,10,15,20,25,40,50,60,80,100 \mathrm{MHz}$ " bandwidth can be used. For $20 \mathrm{MHz}$ bandwidth $19.08 \mathrm{MHz}$ (106 PRBs) with $95.4 \%$ utilization where in LTE only $18 \mathrm{MHz}(100 \mathrm{PRBs})$ utilization .The Inter-Site-Distance (ISD) directly depends on CP length. According to Release-15 maximum ISD is $1.4 \mathrm{~km}$ [8]. This ISD only suitable for small stadiums or malls but not suitable for urban or rural scenarios. Longer ISD obtained by using two negative $\mu$ and extended CP. With negative $\mu$ the ISD goes around 40km. A $60 \mathrm{~km}$ ISD can achieve with a longer CP of $200 \mu \mathrm{s}$ of Release 14. For fixed reception services with large rooftop antennaswith ISD of above $150 \mathrm{~km}$ may be obtained from CPs above $200 \mu \mathrm{s}$. For mobile services with high speed upto $250 \mathrm{~km} / \mathrm{h}$ can be achieved with a CP between $33 \mu \mathrm{s}$ and $200 \mu \mathrm{s}$. The numerologies should be taken appropriately for terrestrial broadcasting. A wide SCS should select for acceptable mobility performance and a very large CP should not be takenfor a single cell or MFN. For moderate speed large CP with $20 \%$ overhead and for static reception or SFN large CP numerologies should be selected as shown in Table-3[7].

Table:3 Different numerologies in NRMBMS [7]

\begin{tabular}{|c|c|c|c|c|c|c|}
\hline $\boldsymbol{\mu}$ & $\begin{array}{c}\Delta \mathbf{f}(\mathbf{K} \\
\mathbf{H z})\end{array}$ & $\begin{array}{c}\mathbf{T}_{\mathbf{U}} \\
(\boldsymbol{\mu} \mathbf{s})\end{array}$ & $\begin{array}{c}\mathbf{T}_{\mathbf{C P}} \\
(\boldsymbol{\mu} \mathbf{s})\end{array}$ & $\begin{array}{c}\text { Overh } \\
\mathbf{e a d} \%\end{array}$ & $\begin{array}{c}\mathbf{I S D}(\mathbf{k}) \\
\mathbf{m})\end{array}$ & $\mathbf{C P}$ \\
\hline 0 & 15 & 66.7 & 4.7 & 7 & 1.4 & NCP \\
\hline 1 & 30 & 33.3 & 2.3 & 7 & 0.7 & NCP \\
\hline 2 & 60 & 16.67 & 1.2 & 7 & 0.36 & NCP \\
\hline 3 & 120 & 8.33 & 0.59 & 7 & 0.175 & NCP \\
\hline 4 & 240 & 4.17 & 0.29 & 7 & 0.087 & NCP \\
\hline 0 & 15 & 66.7 & 16.7 & 20 & 5 & ECP \\
\hline-1 & 7.5 & 133.3 & 33.3 & 20 & 10 & ECP \\
\hline-2 & 3.75 & 266.6 & 66.6 & 20 & 20 & ECP \\
\hline-3 & 1.875 & 333.3 & 133.3 & 20 & 40 & ECP \\
\hline- & 1.25 & 800 & 200 & 20 & 60 & ECP \\
\hline- & 0.625 & 1600 & 400 & 20 & 120 & ECP \\
\hline
\end{tabular}

\section{Performance analysis of 5G-NRMBMS system with twolayerLDM}

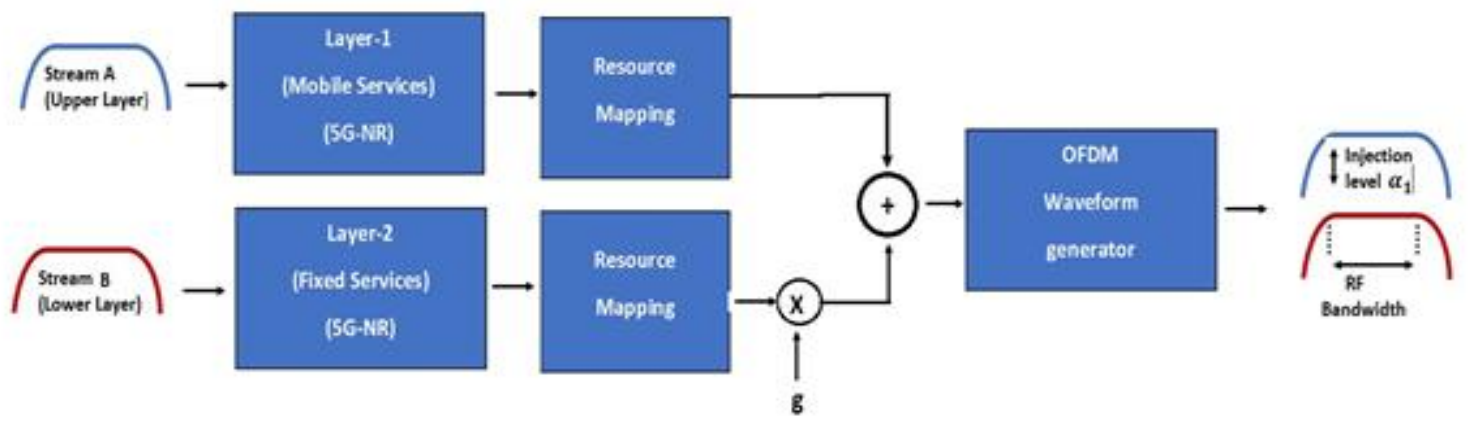




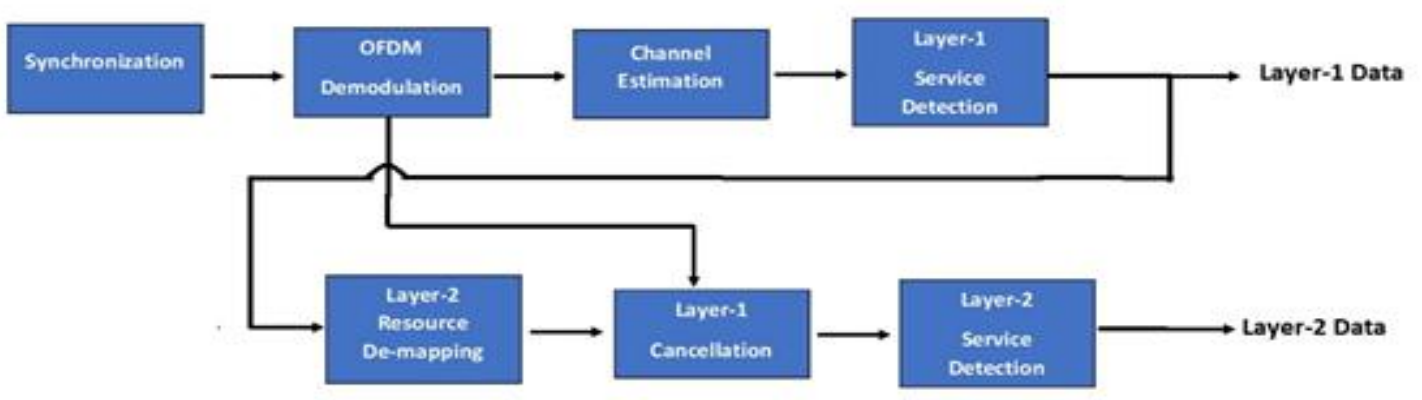

Fig .3 A 5G- NRMBMS transmitter and receiver with 2-layer LDM .

A Two -layer LDM 5G-NRMBMS transmitter and receiver block diagram is shown on above figure-3In the above LDM system both layers are used for broadcasting. The Layer-1 with higher power is for delivering broadcast services to robust mobile/ handheld or indoor receivers with very low SNRs, while the second layer Layer-2 with low power delivers broadcast services to high throughput fixed-receivers with high SNRs. With this LDM system the MIMO technology is also configured with flexible antenna configuration to increase channel performance. For ayerL-1 signal highest -diversity gain (div-MIMO) MIMO technology configured to get robustness signal at low 'SNRs'. For Layer-2 signal highest -“spatial multiplexing gain” (cap-MIMO) configured to get better capacity at high 'SNRs' [9].

Each layer will encode separately with respect to their channel- coding and modulation technique. Then in the resource mapping block the transmission symbol of different layerswill be mapped to a set of RBs (Resource Blocks). The output of the resource mapping block of Layer-1 gives KL-1 symbols for each OFDM symbol and for Layer-2 it gives Kl-2 symbols, whichrepresents the number-of subcarriers. Then the L-1 Symbol and L-2 symbol combined together with a coefficient of $\mathrm{g}$, where $\mathrm{g}$ represents the power level among L-1 and L-2 Layer[1].

$X(k)=X_{L-1}(k)+g \cdot X_{L-2}(k)(2)$

At receiver end, the received signal

$Y(k)=X_{L-1}(k) \cdot H(k)+g \cdot X_{L-2}(k) \cdot H(k)+N_{0}$

Where $\mathrm{H}$ represents the response signal of the $\mathrm{k}_{\mathrm{th}}$ sub-channel. And $\mathrm{N}_{0}$ represents the noise. The decoding of theL-1 signal at the receiver endsameas single layer detection process, where L-2 low-power signal is treated as an interference. But to decode theL-2 signal the receiver needs to decode the L-1 signal then it cancels from received signal, this process is called "successive signal cancellation" (SSC). The L-2 signal represented as [1]

$Y_{L-2}(k)=\frac{1}{g}\left(Y(k)-\tilde{Y}_{L-1}(k)\right)$

$$
=X_{L-2}(k) \cdot H(k)+\frac{1}{g}\left(X_{L-1}(k) \cdot\left(H(k)-\widetilde{H}(k)+N_{0}\right)\right.
$$

$=X_{L-2}(k) \cdot H(k)+\frac{\varepsilon_{L-1}}{g}+\frac{N_{0}}{g}(4)$

where $\widetilde{H}(k)$ is the channel estimate, and L-1 is the residual signal after the SSC. The channel estimator is an important key to get a higher L-2 detection signal. Here L-1 is higher power level then L-2. L-1 is for broadcasting services in SFN mode, in SFN broadcast all transmitters transmitthe same signal simultaneously. The L-1 broadcast service simulated in three different scenariosi.e fixed devices with Ultra-High Definition (UHD) of roof top-antennas, fast moving vehicles with HD mobile services and handheld devices in indoor reception[1].

The layer-1 which is used for mobile services is considered MCS2 modulation and coding scheme where layer-2 for fixed services considering MCS24 modulation and coding scheme. Mobile reception needs minimum SNR , so QPSK modulation with low code rate taken and for high throughput fixed reception 256QAM modulation taken. For mobile reception different factors affect performance, medium speed always gives better performance while high speed affects due to Doppler shift and low speed lack time selectivity. Here mobile reception with a minimum SNR of $6 \mathrm{~dB}$ and reference speed of $60 \mathrm{~km} / \mathrm{h}$ considered. For MCS2 and MCS24 with 
SCS $1.25 \mathrm{kHz}$ gives SNR close to $6 \mathrm{~dB}$ and $20 \mathrm{~dB}$ for mobile and fixed reception respectively. If we compare with LTE FeMBMS below 1dB improvement is provided because of LDPC coding in the physicallayer[1].

Table:4Analysis of Speed and Doppler limit [7]

\begin{tabular}{|l|l|l|l|l|l|l|}
\hline $\boldsymbol{\mu}$ & $\Delta \mathbf{f}(\mathbf{K H z})$ & $\mathbf{T}_{\mathbf{U}}(\boldsymbol{\mu s})$ & $\mathbf{T}_{\mathbf{C P}}(\boldsymbol{\mu s})$ & $\mathbf{D}_{\mathbf{t}}$ & $\begin{array}{l}\text { Doppler } \\
\text { limit(Hz) }\end{array}$ & $\begin{array}{l}\text { Maximum } \\
\mathbf{u s e r} \\
\mathbf{s p e e d} \\
\mathbf{( k m / h )}\end{array}$ \\
\hline 0 & 15 & 66.7 & 16.7 & 3 & 2000 & 3084 \\
\hline-1 & 7.5 & 133.3 & 33.3 & 2 & 1500 & 2133 \\
\hline-2 & 3.75 & 266.6 & 66.6 & 2 & 750 & 1156 \\
\hline-3 & 1.875 & 533.33 & 133.33 & 2 & 375 & 578 \\
\hline- & 1.25 & 800 & 200 & 2 & 250 & 385 \\
\hline- & 0.625 & 1600 & 400 & 2 & 125 & 192 \\
\hline
\end{tabular}

Doppler shifts affect the OFDM system when the user travels with a speed from the transmitter. The Dopplershift is directly related to 'SCS' which affects mobile reception in 'SFN' environment and in large ISD both MFN or single cell also affected while low overhead used.

$$
f_{D}=\frac{1}{2} D_{t}\left(T_{u}+T_{c p}\right) H z(5)
$$

Channel estimation measurement carried out onsubcarriers which arereference signals and it performed on every $\mathrm{D}_{\mathrm{t}}$ thsymbol.The mobile reception performance depends on 'SCS', bandwidth, frequency-band and channel estimation .The Table-4 shows different Doppler limits with different 'SCS'values with different speeds. Figure-4 shows a graph of mobility performances with different numerology. For 'SCS' $15 \mathrm{kHz}$ Doppler-shift goes above $1600 \mathrm{~Hz}$ of speed more than $2400 \mathrm{~km} / \mathrm{h}$. For SCS $1.25 \mathrm{kHz}$ the Doppler shift decreases up to $180 \mathrm{~Hz}$ of speed $280 \mathrm{~km} / \mathrm{h}$.For 'SCS' $625 \mathrm{~Hz}$ giving Doppler shift about $75 \mathrm{~Hz}$ which speed about $115 \mathrm{~km} / \mathrm{h}[7][8]$.

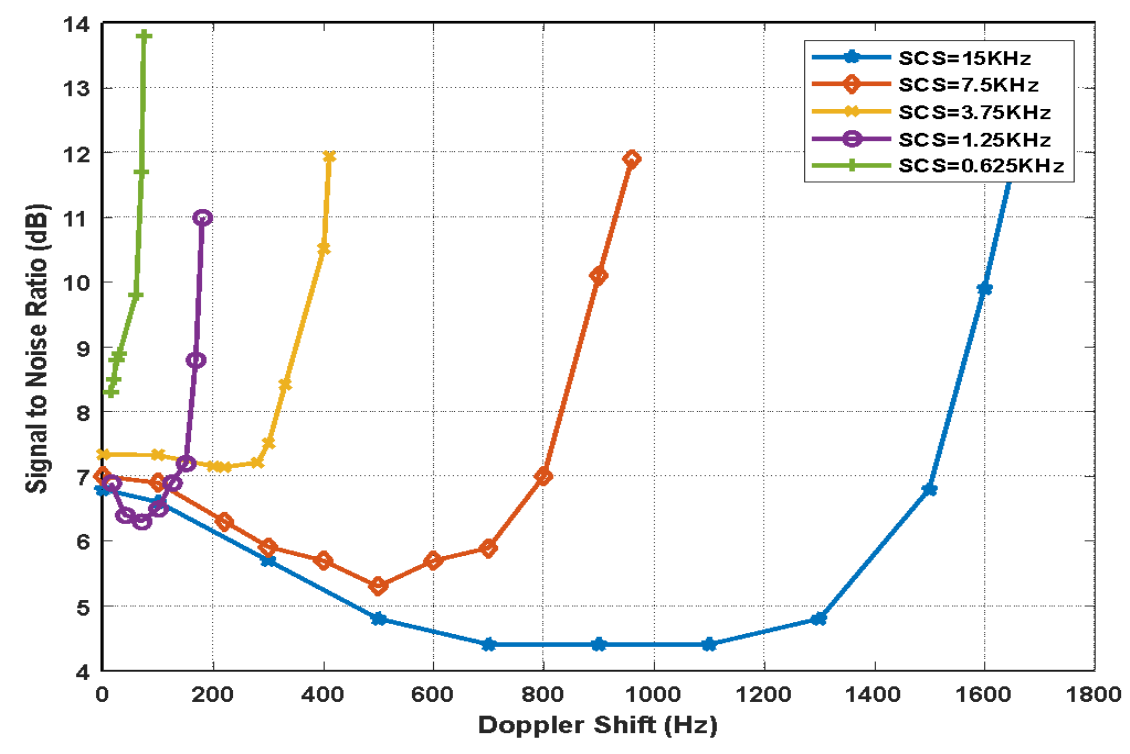

Fig.4 Doppler shift vs. SNR with different SCS of 5G- NRMBMS [7][8]

The coding scheme ofDownlink Control Information (DCI) of 5G-NRMBMS uses polar coding scheme and the size of encoded information depend on Aggregation level(AL)[8]. The use of polar code instead of LDPC reduces the decoding complexity in DCI whereas in LTE Tail- Biting -Convolutional Code(TBCC) used. For different $\mathrm{AL}$ defines for selected DCI format. The scheduler defines appropriate robustness and data rate according to AL. 5G-NR has 'AL' up to 16 whereas for LTE it is only up to 8[7]. The Figure-5 given the performance analysis of SNR vsALup to 16 with different DCI value for fixed-reception analyzed and Figure-6 showing SNR vs AL for mobile receptions. Increasing AL from 8 to 16 reduces $3 \mathrm{~dB}$ SNR requirements approximately than 'LTE' and the problem of detecting the 'CAS' in 'LTE' eliminated [7]. 


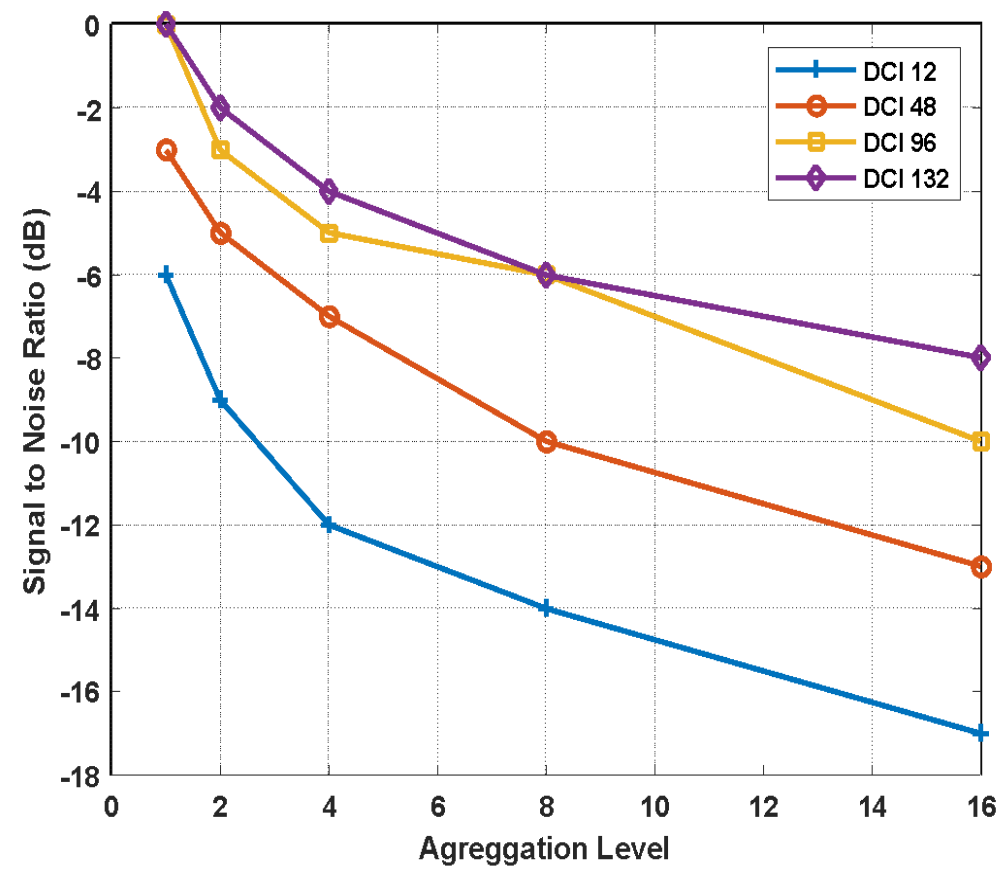

Fig .5 SNR vs Aggregation Level for fixed services

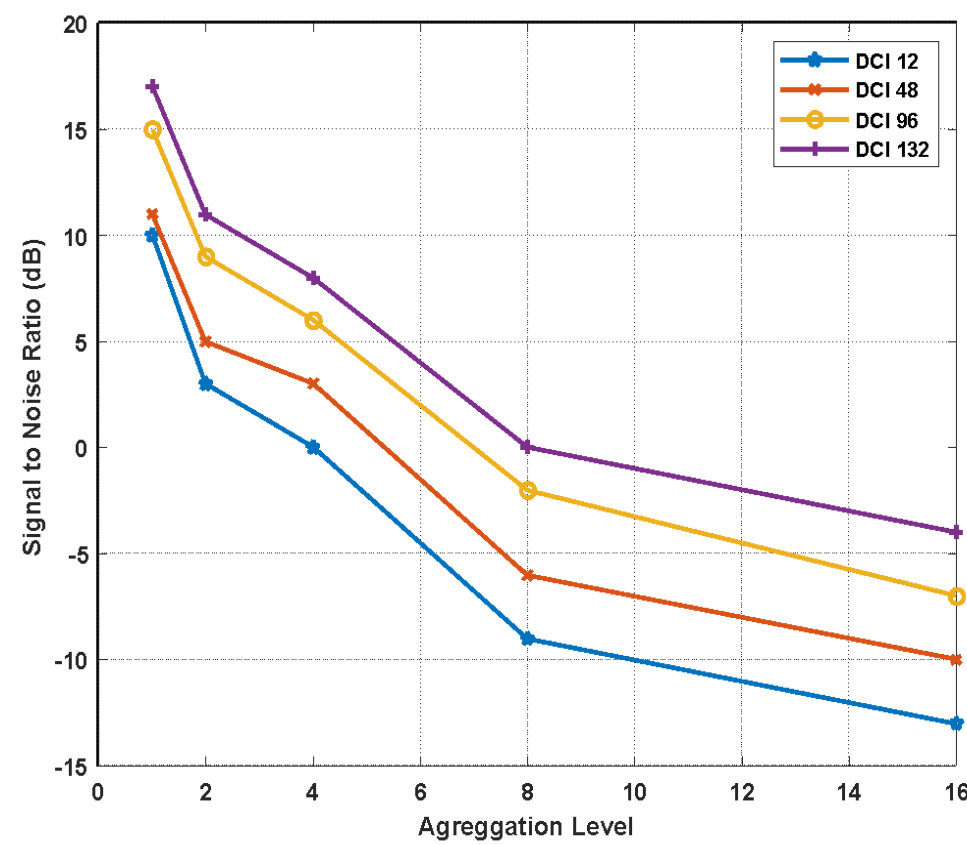

Fig .6 SNR vs Aggregation Level for Mobile services

A LPLT network with 40W effective-radiated power (ERP)and 30m antenna height, whereas for HPHT network with 50kW ERP and 250m antenna height considered in this study. The Figure 7 shows the performance graph of coverage of Fixed reception services with various CP lengths. A CP of $100 \mu$ s is good for the ISD of 5 to $10 \mathrm{~km} .200 \mu \mathrm{s} \mathrm{CP}$ is for more than 70km ISD. 


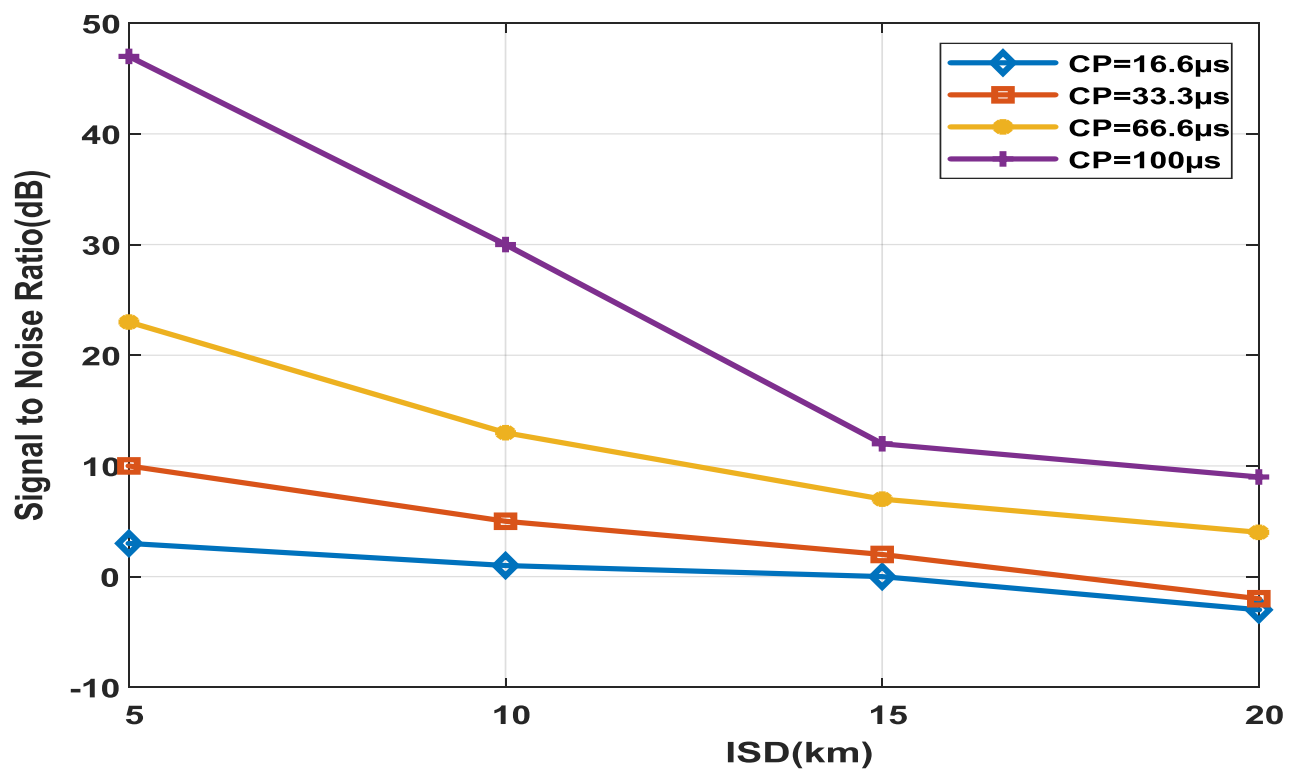

Fig .7 ISD Vs SNR for different CP

\section{Conclusion}

in this study, we have described two-layer LDM and 5G-NR physical layers. Then we integrated these two systems together for use cases of terrestrial broadcasting. we have analyzed with negative numerology and extended cyclic prefix. The signal-to- noise ratio(SNR) in respect of doppler shift, aggregation level and inter-sitedistance(ISD) with $\mu=0,-1,-2,-3$ and extended CP of $-100 \mu \mathrm{s},-200 \mu \mathrm{s},-300 \mu \mathrm{s}$.Use of negative numerology and extended CP values in two- layer LDM 5G-NRMBMS giving better performance for broadcasting services. By using a two -layer LDM increases the spectral efficiency and higher the capacity gain compare to TDM/FDM systems.

\section{References}

Zhang L., W. Li , Y. Wu, Y. Xue, Park E.S.S.I., Lee J.Y., Hur N., Kim H.M.,(2020) Using Non-Orthogonal Multiplexing in 5G-MBMS to Achieve Broadband-Broadcast Convergence With High Spectral Efficiency, IEEE Transactions on Broadcasting, 66(2), pp. 490-502.

Säily M., Estevan C. B., Gimenez F.T., Guo W., Barquero D.G., Mi D.,(2020) 5G Radio Access Network Architecture for Terrestrial Broadcast Services, , IEEE Transactions on Broadcasting, 66(2), pp. $404-415$.

A report on "The 5G Evolution: 3GPP Releases 16-17(2020), Published by 5G Americas, pp 1-54, https://www.5gamericas.org/wp-content/uploads/2020/01/5G-Evolution-3GPP-R16-R17.

Kim H. J., Kwon S., Kim H., Bae J. H., Kim Y. andHur N.,( 2019) Analysis of Channel Capacity for Threelayer Signals in Layered Division Multiplexing System, IEEE International Symposium on Broadband Multimedia Systems and Broadcasting (BMSB), Jeju, Korea (South), pp. 1-4.

Regueiro C., Montalban J., Barrueco J., Velez M., Angueira P., Wu Y., Zhang L., Park S.I., Lee J.Y., Kim H. M., (2016)LDM Core Services Performance in ATSC 3.0, IEEE Transactions on Broadcasting,62(1) , pp 244 -252 .

Zhang L., Wu Y., Li W., Salehian K., Laflèche S., Hong Z., Park S.I., Lee J.Y., Kim H.M., Hur N.,(2018)Using Non-Orthogonal Multiplexing for In-Band Full-Duplex Backhaul for 5G Broadcasting, IEEE 5G World Forum (5GWF), Silicon Valley, CA, pp. 168-173.

Gimenez J.J., Carcel J.L., Fuentes M., Garro E., Elliott S., Vargas D., Menzel C., Barquero D. G..(2019) 5G New Radio for Terrestrial Broadcast:A Forward-Looking Approach for NR-MBMS,IEEE Transactions on Broadcasting, 65(2), pp. 356-368.

Garro E., Fuentes M., Carcel J. L., Chen H., Mi D.; Tesema F., Gimenez J. J., Barquero D.G., (2020)5G Mixed Mode: NR Multicast-Broadcast Services, IEEE Transactions on Broadcasting, 66(2), pp. 390-403,

Zhang L., Wu Y., Li W., Salehian K., Lafleche S., Wang X., Park S. I., Kim H. M., Lee J.Y., Hur N., Angueira P., Montalban J.,(2018) Layered-Division Multiplexing: An Enabling Technology for Multicast/Broadcast Service Delivery in 5G, IEEE Communications Magazine, 56(3), pp. 82-90.

Omri A., Shaqfeh M., Ali A. and Alnuweiri H., ( 2019)Synchronization Procedure in 5G NR Systems, IEEE Access, 7, pp. 41286-41295.. 